\title{
Event reconstruction in NEW using a ML-EM algorithm
}

\author{
Ander Simón Estévez* on behalf of the NEXT Collaboration \\ Instituto de Física Corpuscular, Valencia, Spain \\ E-mail: ander.simondific.uv.es

\section{Christoph Lerche} \\ Institute of Neuroscience and Medicine (INM-4), Forschungszentrum Jülich GmbH, Jülich, \\ Germany \\ E-mail: c.lerchedfz-juelich.de
}

The NEXT collaboration aims to observe the neutrinoless double beta decay $(\beta \beta 0 v)$ of ${ }^{136} \mathrm{Xe}$. If exisiting, the rareness of this decay demands an exceptional background rejection. This can be obtained with an excellent energy resolution, which has been already demonstrated in NEXT prototypes. In addition to this, any additional tool that can improve background rejection is of extreme utility. Neutrinoless double beta decay in xenon gas produces a characteristic topological signal that can be used as an extremely useful extra handle to veto background events. Therefore, for NEXT, the capability of having track reconstruction is of great value. This need for a satisfactory topology reconstruction has led the NEXT Collaboration to implement the Maximum Likelihood Expectation Maximization algorithm (ML-EM) in the data processing scheme. MLEM is a generic iterative algorithm for solving many kinds of inverse problems which has never been applied before to a time projection chamber. Early results and performance of the method in the collaboration current running detector, NEW, shows promising track reconstruction while offering a resolution better than $0.5 \%$ for energy depostions of energy equal to the $\mathrm{Q}_{\beta \beta}$ value for ${ }^{136} \mathrm{Xe}$.

38th International Conference on High Energy Physics

3-10 August 2016

Chicago, USA

${ }^{*}$ Speaker. 


\section{The NEXT experiment}

Neutrinoless double beta decay is a hypothetical process where two neutrons of a nuclei transform into two protons emitting only two electrons and no neutrinos. The existance of this decay would imply that neutrinos are Majorana particles [1].

The experimental signature of this event consists in a fixed energy deposition equal to the energy difference between the decaying nucleus and its daughter. In addition, if produced in high pressure gas, the energy deposition will occur along an erratic path with two end-points of higher energy. This kind of behaviour in gas is unique to double beta decay.

Current experimental measurements estimate the half life of this decay in the xenon isotope to be longer than $10^{25}$ years. Such a rareness implies that background rejection is key in the performance of the experiments. Good energy resolution plus other ways to improve the rejection of background are essential requirements for any $\beta \beta 0 \mathrm{v}$ experiment.

NEXT [2] will look for the $\beta \beta 0 v$ of ${ }^{136} \mathrm{Xe}$ in a high-pressure gaseous xenon (HPGXe) time projection chamber with an intense electric field region to produce light through electroluminiscence. This serves as the amplification method. It is designed to offer outstanding energy resolution for a xenon experiment while having the capability of analyzing the track shape of the events which can be used as a powerful tool for background discrimination. This is possible due to the assymetric detector design, with one plane composed of PMTs and optimized for energy measurements while the other is equipped with SiPMs that provide pixel information.

At the moment, the collaboration is comissioning its largest detector so far, NEXT-White or NEW, which will provide a precise background model for the experiment. In addition it will serve as a demonstrator of the performance of the technology in larger volumes than prototypes.

\section{Maximum Likelihood Expectation Maximization}

Maximum Likelihood Estimation (MLE) is a method suitable for solving a vast variety of inverse problems. The underlying idea behind it is to maximise the likelihood of a given statistical model that describes the forward problem. Having a given response, the parameters that maximize the likelihood of the model provide the most probable cause of the response. This method is especially useful in complex inverse problems. This is the case of NEXT. However, obtaining a solution is not immediate if not impossible for several cases.

One can use the log-likelihood function instead of the likelihood which expressed mathematically would be:

$$
x_{M L}=\arg \max \log \mathscr{L}(x \mid y),
$$

where $x_{M L}$ is the ML estimate, $\mathscr{L}$ is the likelihood function, $y$ is the vector of observations and $x$ is the vector of unknown parameters. The log-likelihood to be maximized depends on the underlying 
model. For a Poisson process, one can derive the following log-likelihood function:

$$
\begin{aligned}
\log \mathscr{L}(x \mid y) & =\log \prod_{l}^{m} P\left(y_{l} \mid x\right) \\
& =\log \prod_{l}^{m} \frac{e^{-\left.A x\right|_{l}}\left(\left.A x\right|_{l}\right)^{y_{l}}}{y_{l} !} \\
& =-\sum_{l}^{m}\left(\left.A x\right|_{l}-y_{l} \log \left(\left.A x\right|_{l}\right)+\log \left(y_{l} !\right),\right.
\end{aligned}
$$

where $m$ is the dimension of $y$ and $A$ is the transformation matrix applied to $x$ to result in $y$. Even with the use of the log-likelihood, problems that allow for explicit analytical solutions are usually extremely rare. Therefore, iterative approaches have to be implemented. In this case the Expectation Maximization can be used. For this, hidden variables are introduced purely as a mathematical tool. They are implemented in a way that the maximization is considerably simplified.

$$
\mathscr{L}(x \mid y)=P(y \mid x)=\sum_{z} P(y, z \mid x),
$$

where $z$ is the vector of latent variables and $P(y, z \mid x)$ is the marginal probability of the observed data. If we know the value of the parameters $x$, we can find the value of the latent variables $z$ by maximizing the log-likelihood over all possible values of $z$. Conversely, if we know the value of the latent variables $z$, we can find an estimate of the parameters $x$ fairly easily by simply grouping the observed data points according to the value of the associated latent variable. This suggests an iterative algorithm, in the case where both $x$ and $z$ are unknown. The algorithm works as follows:

1. Initialize the parameters $x$ to an initial value.

2. Expectation step (E-step): compute the best value for $z$ given these parameter values.

3. Maximization step (M-step): Use the values of $z$ to compute a better estimate for $x$.

4. Iterate steps 2 and 3 until convergence.

\section{Applying ML-EM in NEXT}

The ML-EM algorithm can be applied to several problems and, specifically, it has been broadly used in processes of Poisson nature. This is exactly the case of NEXT where scintillation and photon counting are the underlying processes and, therefore, can be described using eq. (2.2). If then Expectation Maximization is applied the following formula can be obtained [3, chap. 5.3]:

$$
\lambda_{m}(v)=\frac{\lambda_{m-1}(v)}{\sum_{d} P(v, d)} \sum_{d} \frac{n(d) P(v, d)}{\sum_{v}^{\prime} \lambda_{m-1}\left(v^{\prime}\right) P\left(v^{\prime}, d\right)},
$$

where $\lambda(v)$ is the charge deposited in voxel $v$ being $v$ a volume unit in which the active volume of the TPC is discretized, $P(v, d)$ is the probability of detection by the detector $d$ of a photon produced due to charge deposited in $v$ and $n(d)$ is the number of photoelectrons produced in $d$.

Therefore, to use ML-EM in NEXT a voxelization of the active volume of the detector is necessary. In addition to that, the probabilities $P(v, d)$ must be computed. This is done using a 
MC simulation which serves as a way of obtaining a parametrization function that convolves all the effects that can have an impact on the signal.

The observables are the sensor response in both the tracking and energy plane of the TPC. This signal is also used as the seed for the first iteration, $\lambda_{0}(v)$. In order to avoid any prior bias, initial charge of voxels is set as a uniform distribution of the total number of photoelectrons produced at the PMTs. The output is the collections of voxels that represent the charge distribution inside the chamber, therefore it's the track produced by the event (Fig. 1a).

At the same time, the addition of the charge of all voxels gives an estimate for the event energy (Fig. 1b). ML-EM then provides with just one method a way to obtain both the corrected energy and the track of any event with outstanding performance for both tasks.
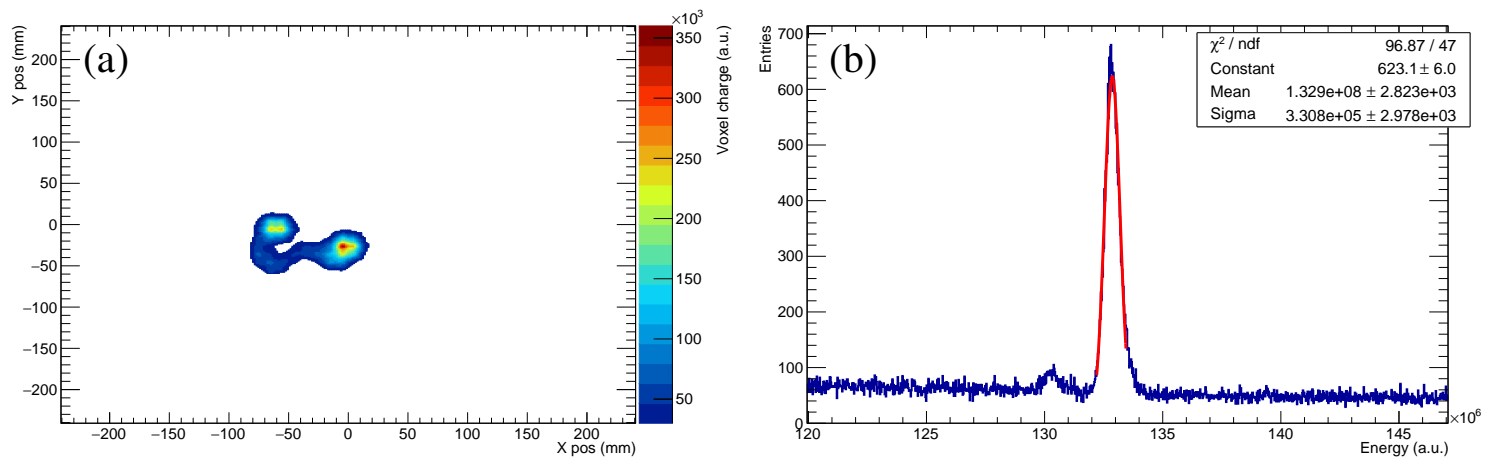

Figure 1: (a) Characteristic track of a simulated $\beta \beta 0 v$ event in NEW detector reconstructed using ML-EM. (b) Reconstructed energy of the $1.6 \mathrm{MeV}$ peak of ${ }^{208} \mathrm{Tl}$. Fit shows a $0.58 \%$ FWHM energy resolution. It extrapolates to $0.47 \%$ at $\mathrm{Q}_{\beta \beta}$.

Currently, only a bidimensional parameterization is used. This implies that all the longitudinal position dependent effects like diffusion or attachment are not considered. Even so, results obtained are promising and satisfactory. In future work, a more detailed study of the topology response of the method and the implementation of a full 3D parameterization of the detection probabilities will be included.

\section{References}

[1] E. Majorana, A symmetric theory of electrons and positrons, Nuovo Cimento 5, 1937: p. 171.

[2] NEXT collaboration, J. Martín-Albo et al., Sensitivity of NEXT-100 to neutrinoless double beta decay, Journal of High Energy Physics, 2016: p. 159, arxiv:1511.09246.

[3] Natterer, Frank and Wubbeling, Frank. Mathematical Methods in Image Reconstruction, 2001, ISBN 0-89871-472-9, Society for Industrial and Applied Mathematics, Philadelphia, PA, USA.

\section{Acknowledgement}

The NEXT Collaboration acknowledges support from the ERC under the Advanced Grant 339787-NEXT; the Ministerio de Economía y Competitividad of Spain and FEDER under grants CSD2008-0037 (CUP), FIS2014-53371-C04 and SEV-2014-0398; GVA under grant PROMETEO/2016/120. Fermilab is operated by Fermi Research Alliance, LLC under Contract No. DEAC02-07CH11359 with the United States Department of Energy. 